\title{
Three-Dimensional Through-Space/Through-Bond Delocalization in Cyclophane Systems: A Molecule-in-Molecule Approach
}

\author{
Hsin-Chieh Lin and Bih-Yaw Jin* \\ Department of Chemistry, National Taiwan University, Taipei, Taiwan 106
}

Received: August 7, 2007; In Final Form: January 14, 2008

\begin{abstract}
In this article, we propose a simple strategy to identify the nature of excitonic couplings in a series of cyclophanedienes based on the "molecule-in-molecule" (MIM) theory. The contributions of charge-transfer (CT) exciton, unavailable by the commonly used supermolecular approach due to the inadequate basis set, can be unambiguously identified within this methodology. Combining the CT contributions calculated on the cyclophanedienes and the corresponding hypothetical molecules with tethers removed, one can infer the information on the relative importance of through-bond and through-space contributions in the low-lying excited states. Particularly, we discovered that the tether effect for the meta-linkage cyclophanedienes is crucial, whereas those for para-linkage cyclophanedienes are vanishingly small. The changes in the coupling between two moieties for both the six-membered meta-linkage and five-membered cyclophanedienes arise primarily from an increase in the through-bond charge-transfer component of the coupling $(>70 \%)$. Within the MIM model, the delocalization pathway of the dimer in the excited state can be explained quantitatively by a CT exciton, which differs from the approach based on the conventional orbital interaction analysis.
\end{abstract}

In this article we will investigate theoretically the nature of the chromophore-chromophore interactions that play a critical role in designing optoelectronic materials. ${ }^{1-9}$ The progress in our understanding of chromophore-chromophore interaction has been hindered due to the difficulty in controlling the relative arrangements for different molecules in amorphous organic solid. Cyclophane molecules are promising candidates for designing a new class of organic-based electro-optical devices because the chromophore-chromophore interactions with welldefined distance and orientation could be easily controlled. ${ }^{2}$ Recently, bichromophoric and multichromophoric cyclophanes have been studied for organic solids, ${ }^{3}$ biosensors, ${ }^{4}$ eletrocyclic reactions, ${ }^{5}$ two-photon absorption, ${ }^{6}$ mixed-valence systems, ${ }^{7}$ and nonlinear optical materials. ${ }^{8,9}$ Particularly, Bazan et al. have exploited the advantage of well-defined three-dimensional structures in cyclophanes that contains [2.2]paracyclophane core and found their potential applications in designing the organic nonlinear optical materials. ${ }^{8}$ More recently, it has been shown that chromophores with twisted $\pi$-electron systems in a cyclophane system having five-membered hetero-aromatic rings as electron donors and a bridging double bond as electron acceptor exhibit molecular hyperpolarizability with exceptionally high $\mu \beta$ values. ${ }^{9}$ Interestingly, quantum-chemical calculations suggest that these cyclophane architectures with one or two unsaturated bridging double bonds reveal intramolecular charge-transfer characteristics in the lowest excited state. ${ }^{9,10}$ Accordingly, it is intriguing to study the nature of the excited-state delocalization in detail for these bichromophoric cyclophanes with unsaturated tethers.

In the present work, we roughly distinguished the bichromophoric cyclophanes into three classes according to the dihedral angles between chromophores and the tethered double bonds as shown in Scheme 1. For molecules belonging to class I, the $\pi$-orbitals on the chromophore are almost perpendicular

\footnotetext{
* Corresponding author. E-mail: byjin@ntu.edu.tw.
}

SCHEME 1: Three Types of Dimeric Cyclophandienes with Various Dihedral Angles between Chromophores and Tethered Double Bonds
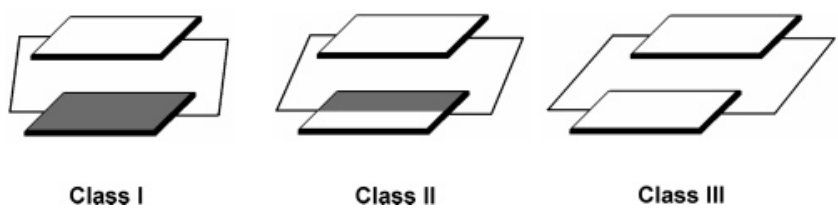

SCHEME 2

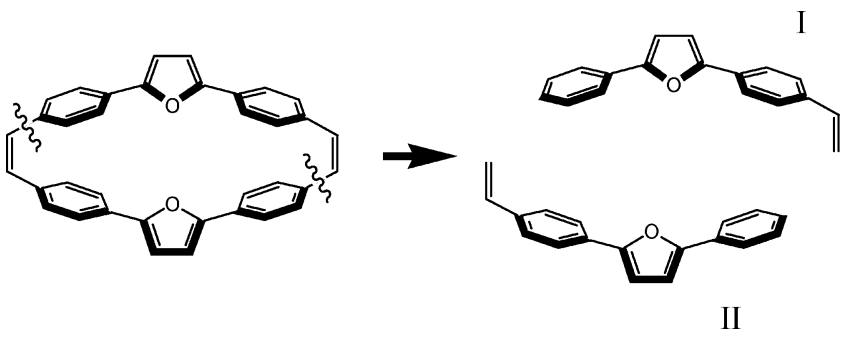

to $\pi$-orbitals on the tethers, and the pathway for this kind of excited-state delocalization is clearly through-space. On the other hand, for molecules in class III, the $\pi$-orbitals of the chromophore are parallel to those of tethers and the delocalization pathway should be through-bond. The most interesting behavior appears in molecules belonging to the class II, where both kinds of delocalization pathways contribute to the transannular interaction between chromophores. It is an interesting synthetic challenge to construct cyclophane molecules belonging to the class II. Incorporation of five-membered heteroaromatic rings into teraryl-cyclophane system could lead to the dihedral angle between chromophore and tether about $40-45^{\circ}$. We have previously synthesized the furan-containing cyclophanes containing saturated and unsaturated tethers. ${ }^{9}$ Cyclophanes with double-bonded tether could have a new low-energy band in the absorption spectrum but the corresponding saturated cyclophane 
with only single bond tether did not. These results suggest that the three-dimensional cyclophene reveals a new charge resonance (CR) band. The delocalization pathway of the new CR band could come from through-bond and/or through-space delocalization. The main issue we want to address here is the delocalization pathway of the class II cyclophanes.

The dimer wavefunction, as expressed by eq 1 , has four resonance forms, two local excitations (Frenkel excitons) and two charge-transfer excitations (CT excitons).

$$
\begin{aligned}
& \psi=c_{1}\left[\psi\left(M^{*} M\right) \pm \psi\left(M M^{*}\right)\right]+ \\
& c_{2}\left[\psi\left(M^{+} M^{-}\right) \pm \psi\left(M^{-} M^{+}\right)\right]
\end{aligned}
$$

Here, we suggest that the weight of the CT exciton in the wavefunction could be used to determine the nature of delocalization in the cyclophane excitations. However, the widespread theoretical model, the supermolecular approach, ${ }^{11}$ in studying cyclophanes could not directly provide this critical information because of the inadequacy of the basis set construction based on completely delocalized molecular orbitals obtained by the Hartree-Fock calculations. In this work, the "moleculein-molecule" (MIM) calculation based on the construction of CI matrix with localized molecular orbitals will be exploited to address the nature of excited states in the class II molecules. Furthermore, we also propose a simplified four-state MIM model to extract electronic coupling from the calculation based on the supermolecular calculation. To the best of our knowledge, the nature of excited-state delocalization of cyclophane systems with the double-bonded tether has not been studied in detail; therefore, we try to find out the influence of the $\pi$-bond tether on the CR band in the lowest-lying excited state by using the truncated MIM method. As discussed above, the wavefunction of an excited state can be divided into two parts: local-exciton and CT-exciton contributions. The contribution of the CT exciton could be enhanced in double-layered cyclophane systems when the distances between two chromophores are close in space, and therefore, one can take advantage of this key information to determine the delocalization pathway by the MIM method.

\section{Molecule-In-Molecule Model}

The "molecule-in-molecule" (MIM) theory was originally developed by Longuet-Higgins and Murrel for studying excited states of the biphenyl systems based on the fragment molecular orbitals localized on each phenyl group. ${ }^{12}$ Warshel and Parson extended this model to investigate the spectroscopic properties of photosynthetic reaction centers ${ }^{13}$ and molecular crystals. ${ }^{14}$ For cyclophanes, this method is likely to be the most informative if we aim to obtain the dimeric properties from monomeric parameters. To construct the MIM Hamiltonian matrix, we disconnect the $\mathrm{C}-\mathrm{C}$ chemical bonds between the tethers and the adjacent benzene rings as shown in Scheme 2. Cyclophanes can be regarded as the union of two constructing fragments. As such, we could construct the cyclophane MIM Hamiltonian from two non-interacting monomers (fragments), in which the four diagonal blocks stand for localized Frenkel-exciton, CTexciton, hole-transfer, and electron-transfer subspaces, and the off-diagonal blocks represent the interaction among them. The localized Frenkel-exciton subspace will be first partially diagonalized by an intermediate transformation, ${ }^{13}$ such that the wavefunctions of whole cyclophane can be written in terms of the superposition of Frenkel and CT exciton instead of the local configuration-state functions. The excited-state wavefunctions of the monomer were obtained by the Pariser-Parr-Pople
Hamiltonian by considering the direction cosine in resonance integrals to study nonplanar conjugated systems (see the Supporting Information). This method has been used extensively in the studies of conjugated molecules, and it has given reliable results for spectroscopic and other linear or nonlinear optical properties in comparison with experimental results ${ }^{13,14}$ and computationally highly extensive ab initio calculations. ${ }^{15}$

\section{Cyclophane Wave Functions}

The localized molecular orbitals of fragment I and II in cyclophane molecules,

$$
\phi_{n}^{\mathrm{I}}=\sum_{p}^{\mathrm{I}} C_{n, p} \chi_{p}^{\mathrm{I}} \quad \text { and } \quad \phi_{m}^{\mathrm{II}}=\sum_{q}^{I I} C_{m, q} \chi_{q}^{\mathrm{II}}
$$

are obtained by solving the corresponding Hartree-Fock equations of each isolated fragments (see the Supporting Information). The basis function $\chi_{p}$ and $\chi_{q}$ are taken to be the $\mathrm{p}_{z}$ atomic orbital on atoms $p$ and $q$, respectively. The interaction between the two moieties in the cyclophane is introduced at the level of configuration interaction by constructing a CI matrix (A) with the matrix elements given by

$$
\begin{aligned}
\mathrm{A}_{\mathrm{N}, \mathrm{M}}^{\mathrm{phane}}=\delta_{n_{\mathrm{a}}, m_{\mathrm{a}}} F_{n_{\mathrm{r}}, m_{\mathrm{r}}}-\delta_{n_{\mathrm{r}}, m_{\mathrm{r}}} F_{n_{\mathrm{a}}, m_{\mathrm{a}}}- \\
\qquad m_{\mathrm{a}} n_{\mathrm{r}}\left|n_{\mathrm{a}} m_{\mathrm{r}}\right\rangle+2\left\langle m_{\mathrm{a}} n_{\mathrm{r}} \mid m_{\mathrm{r}} n_{\mathrm{a}}\right\rangle
\end{aligned}
$$

where $\mathrm{N}$ and $\mathrm{M}$ stand for singly excited configuration functions. The indices, a and $\mathrm{r}$, indicate the occupied and unoccupied molecular orbitals, respectively. The matrix elements of $\mathbf{F}$ matrix are given by eq $3 b$, and the two-electron integrals can be evaluated by using eq $3 \mathrm{c}$ within the zero-differential overlap (ZDO) approximation.

$$
\begin{aligned}
\mathbf{F}_{n j, m k} & =\sum_{p}^{I} \sum_{q}^{I I} C_{n j, p} C_{m k, q}\left\langle\chi_{p}\left|H_{p, q}^{\mathrm{core}}\right| \chi_{q}\right\rangle \\
& =\sum_{p}^{I} \sum_{q}^{I I} C_{n j, p} C_{m k, q} \beta_{p, q}
\end{aligned}
$$

$$
\begin{aligned}
\left\langle m_{\mathrm{a}} n_{\mathrm{r}} \mid n_{\mathrm{a}} m_{\mathrm{r}}\right\rangle & =\iint \phi_{m_{\mathrm{a}}}^{\mathrm{II}}(1) \phi_{n_{\mathrm{r}}}^{\mathrm{I}}(2)\left(1 / r_{12}\right) \phi_{n_{\mathrm{a}}}^{\mathrm{I}}(1) \phi_{m_{\mathrm{r}}}^{\mathrm{II}}(2) \mathrm{d} \tau_{1} \mathrm{~d} \tau_{2} \\
& \approx \sum_{p}^{\mathrm{I}} \sum_{q}^{\mathrm{II}} C_{m_{\mathrm{a}}, q} C_{n_{\mathrm{r}}, p} C_{n_{\mathrm{a}}, p} C_{m_{\mathrm{r}}, q} \gamma_{p, q}
\end{aligned}
$$

Subsequently, we apply the intermediate transformations on $\mathbf{A}^{\text {phane }}$ matrix such that the local-exciton subspaces are diagonalized in the local-exciton wavefunctions,

$$
\Psi_{i}^{\mathrm{I}}=\sum_{\mathrm{N}}^{\mathrm{I}} \xi_{i, \mathrm{~N}}{ }^{1} \psi_{\mathrm{N}}^{\mathrm{I} \rightarrow \mathrm{I}} \quad \text { and } \quad \Psi_{k}^{\mathrm{II}}=\sum_{\mathrm{M}}^{\mathrm{II}} \xi_{k, \mathrm{M}}{ }^{1} \psi_{\mathrm{M}}^{\mathrm{II} \rightarrow \mathrm{II}}
$$

where $\psi_{\mathbf{N}}^{\mathrm{I} \rightarrow \mathrm{I}}$ and $\psi_{\mathrm{N}}^{\mathrm{II} \rightarrow \mathrm{II}}$ are the local excited configurations involving removing one electron from an occupied to an unoccupied orbitals of fragments I and II, respectively.

The CI expansion coefficients are obtained by solving the CI matrix (see the Supporting Information). The excitoninteraction matrix can be described by Columbic, exchange and penetration terms ${ }^{16}$ where the exchange term is zero when ZDO approximation is applied. Penetration is a short-range process, which scales as the overlap between the two-chromophore orbitals (i.e., decay exponentially with distance). The conjugated 
SCHEME 3: Truncated MIM Model with Symmetry Constraint

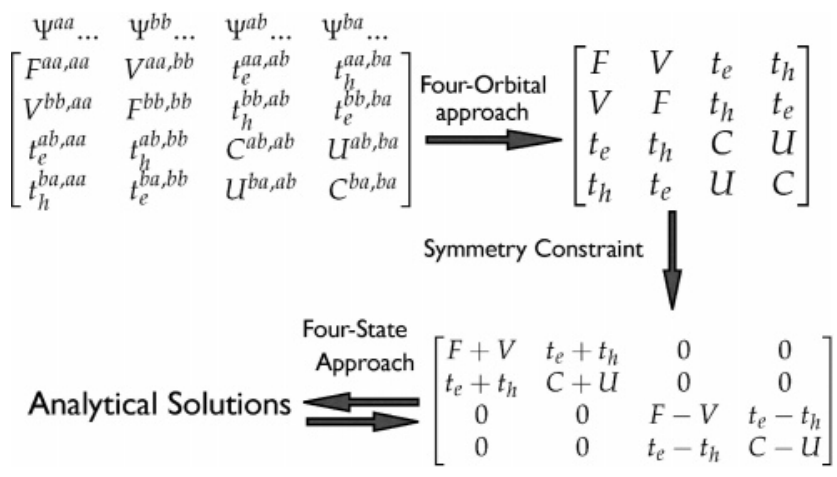

chromophores with interchain interaction or intrachain chainend interaction have been examined, and the Columbic interaction is good enough for describing the energy-transfer process. ${ }^{16 \mathrm{~b}}$ Therefore, here, we consider the most important Columbic term as given in

$$
\begin{aligned}
& { }^{\mathrm{U}} \mathbf{A}_{i, k}^{\mathrm{I}, \mathrm{II}}=\left\langle\Psi_{i}^{\mathrm{I}}|H| \Psi_{k}^{\mathrm{II}}\right\rangle= \\
& 2 \sum_{N}^{\mathrm{I}} \sum_{M}^{\mathrm{II}} \sum_{p}^{\mathrm{I}} \sum_{q}^{\mathrm{II}} \xi_{i, N} \xi_{k, M} C_{m_{\mathrm{a}}, p} C_{m_{\mathrm{r}}, p} C_{n_{\mathrm{a}}, q} C_{n_{\mathrm{r}}, q} \gamma_{p, q}
\end{aligned}
$$

The remaining submatrices in the MIM model can be estimated (see the Supporting Information).

\section{Truncated MIM Method}

It is hard to obtain the weight of charge-transfer (CT) exciton in a typical excited-state quantum chemical calculation based on commercial packages. In this section, we introduce a simplified four-state method based on the truncated MIM Hamiltonian as shown in Scheme 3, which allows us to obtain the relative contributions of the Frenkel (local) and chargetransfer (CT) excitons in the supermolecular calculations. The truncated MIM Hamiltonian at SCI level of a molecular dimer such as a cyclophane is constructed as a minimal CI matrix, which includes only the most important transitions (two local and two CT excitons) among four essential frontier orbitals. The diagonal matrix elements $\mathrm{F}$ and $\mathrm{C}$ are energies to create a Frenkel and a CT exciton, respectively. There are two kinds of the off-diagonal matrix elements. First, the exciton-exciton interaction among two localized Frenkel (CT) exciton, $V$ and $U$, is responsible for the exciton delocalization. Second, the matrix elements, $t_{\mathrm{h}}$ and $t_{\mathrm{e}}$, are the hole and electron-transfer coupling, respectively. The truncated MIM Hamiltonian can be block-diagonalized with the $C_{2}$ symmetry of these cyclophanes, and then the analytical solutions can be obtained by solving the resulting quadratic equations. Combining the energies of excited states calculated by quantum chemical methods (INDO/ $\mathrm{S}$, TD-DFT and so on) with the analytical solutions, this fourstate approach can be used to estimate the matrix element $F$, $C, V$, and $U$ in the effective MIM Hamiltonian. For the four excited states, characterized by the symmetries of the configuration description, only two sets of the configurations are expected: the first set includes the HOMO $\rightarrow$ LUMO and $\mathrm{HOMO}-1 \rightarrow$ LUMO +1 transitions $\left(E_{\mathrm{f} 1}\right.$ and $E_{\mathrm{f} 2}$ in the order of increasing energy), and the other set contains the HOMO $\rightarrow$ $\mathrm{LUMO}+1$ and HOMO $-1 \rightarrow$ LUMO transitions. $\left(E_{\mathrm{a} 1}\right.$ and $\left.E_{\mathrm{a} 2}\right)$ The estimation of the electronic coupling can be obtained by a number of the computational techniques. ${ }^{17}$ One widespread approach is to use the Koopmans' theory ${ }^{18}$ and to estimate the transfer integral for holes (electrons) as half the splitting of the HOMO (LUMO) levels. ${ }^{19}$ The applicability of Koopmans' theory was in good agreement with many other ab initio calculations. ${ }^{20}$ Therefore, we used this method to estimate the hole (electron)-transfer matrix elements for model cyclophanedienes. To evaluate the correct coupling, we have checked the electron distribution in the essential four orbitals by the symmetric and antisymmetric combinations of the single teraryl chromophores.

It is noteworthy that this method still remains true in cyclophanediene systems when hyperconjugating interaction between chromophores and tethers is relatively weak that we will discuss in detail in the text. Within this methodology, the matrix elements of CT exciton $(C)$ and its coupling $(U)$ in this four-state MIM model can be found as

$$
\begin{aligned}
& C=(1 / 2)(\Gamma+\Omega) \\
& U=(1 / 2)(\Gamma-\Omega)
\end{aligned}
$$

where $\Gamma$ and $\Omega$ are

$$
\begin{aligned}
& \Gamma=(1 / 2)\left[\left(E_{\mathrm{a} 1}+E_{\mathrm{a} 2}\right)+\sqrt{\left(E_{\mathrm{a} 1}-E_{\mathrm{a} 2}\right)^{2}-4\left(t_{\mathrm{e}}+t_{\mathrm{h}}\right)^{2}}\right] \\
& \Omega=(1 / 2)\left[\left(E_{\mathrm{f} 1}+E_{\mathrm{f} 2}\right)+\sqrt{\left(E_{\mathrm{f} 1}-E_{\mathrm{f} 2}\right)^{2}-4\left(t_{\mathrm{e}}-t_{\mathrm{h}}\right)^{2}}\right]
\end{aligned}
$$

Furthermore, the Frenkel exciton $(F)$ and exciton coupling $(V)$ can also be determined as

$$
\begin{aligned}
& F=(1 / 2)\left(E_{\mathrm{a} 1}+E_{\mathrm{a} 2}+E_{\mathrm{f} 1}+E_{\mathrm{f} 2}\right)-C \\
& V=(1 / 2)\left(E_{\mathrm{a} 1}+E_{\mathrm{a} 2}-E_{\mathrm{f} 1}-E_{\mathrm{f} 2}\right)-U
\end{aligned}
$$

Combining transfer integrals, $t_{\mathrm{e}}$ and $t_{\mathrm{h}}$, with the energies, $E_{\mathrm{a} 1}$, $E_{\mathrm{a} 2}, E_{\mathrm{f} 1}$, and $E_{\mathrm{f} 2}$, of four excited states obtained from the supermolecular SCI calculation, eqs 6-11 can be used to determine matrix elements $F, U, C$, and $V$. With all these matrix elements estimated, we can calculate the $\mathrm{CT}$ contributions by this simplified model.

Computational Methodology. The ground-state structure of cyclophanes were performed on the semiempirical HartreeFock Austin Model 1 (AM1) method ${ }^{21}$ and the optimized geometries obtained were usually in good agreement with the structures by the X-ray scattering. ${ }^{22}$ In our study, we optimized the model cyclophanes by both the semiempirical AM1 method and the density functional theory (DFT) method to prevent the potential artifact of the computational methods adopted. The density functional theory (DFT) method, we employed the B3LYP functional, where Becke's three-parameter hybrid exchange functional is combined with the Lee-Yang-Parr correlation functional. ${ }^{23}$ All DFT calculations were carried out with the $6-31 \mathrm{G}^{* *}$ split valence plus polarization basis set. ${ }^{24}$ According to other reports, this method can provide reasonable cyclophane structures compared with X-ray data ${ }^{25}$ and other ab initio results. ${ }^{26}$ The excitation energies and electronic structures were calculated by the single configuration-interaction $(\mathrm{SCI})^{27}$ scheme with all occupied and unoccupied $\pi$-levels included within the semiempirical intermediate neglect of differential overlap (INDO) model, ${ }^{28}$ as parametrized by Zerner and coworkers. ${ }^{29}$ The all-valence INDO/S method reasonably provides consistent results of excitation energies and molecular orbitals energies in comparison with the UV/vis spectrum ${ }^{30}$ and UPS ${ }^{31}$ experimental observations of conjugated molecules, respectively. 


\section{SCHEME 4: Molecular Structures of Cyclophandiene 1-4}

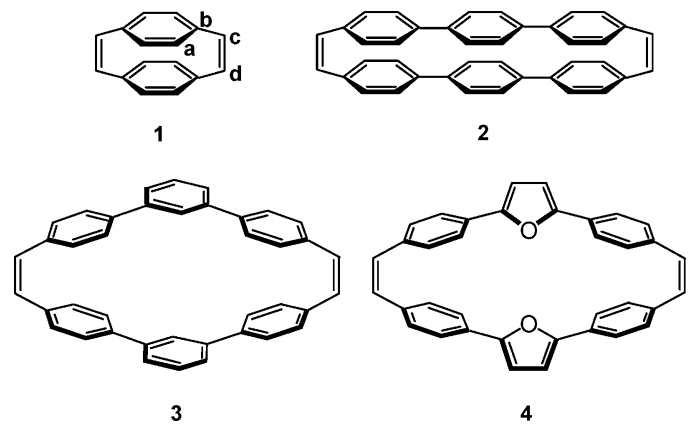

Through-Space/Through-Bond Delocalization. MIM (Full SCI and Truncated N-Orbital Model). The two benzene moieties in the AM1 optimized geometry of molecule $\mathbf{1}$ are almost lying in a near face-to-face arrangement with dihedral angle between the tethered double bond and the benzene moiety about $83^{\circ}\left(\mathrm{C}_{a} \mathrm{C}_{b}-\mathrm{C}_{c} \mathrm{C}_{d}\right.$ in Scheme 4). Hence, this molecule is an ideal model compound belonging to class I. Similarly, the AM1-optimized geometry of molecule $\mathbf{2}$ also shows a slightly tilted cofacial arrangement of the two teraryl chromophores with a dihedral angle around $75^{\circ}$. Note that the aromatic rings in molecule $\mathbf{1}$ and $\mathbf{2}$ are both connected in para-linkage positions with the geometries belonging to the class I. On the other hand, the molecules with the meta-linkage have qualitatively different optimized geometries because their dihedral angles are reduced significantly. Hence, this kind of molecule belongs to class II. For instance, the AM1-optimized dihedral angle for molecule 3 is about $45^{\circ}$. In this regard, introducing meta-linkage aromatic moieties into cyclophane may lead to molecular systems for studying class II models. Incorporation of five-membered heteroaromatics such as furan, thiophene, and pyrrole can also be viewed as a particular case of meta-linkage cyclophane. The main difference between the meta-benzene and five-membered heteroaromatics is that the meta-benzene would interrupt the conjugation at two ends, and the five-membered heteroaromatics retain efficient conjugation among them. In this article, the furan-containing [2.2]cyclophanediene $\mathbf{4}$ was employed as a model compound for class II. The AM1-optimized dihedral angle for this molecule is $45^{\circ}$, which is quite close to the value obtained from DFT-optimized geometry, $40^{\circ}$, indicating that the cyclophanediene $\mathbf{4}$ could be considered as class II molecule.

The nature of delocalization pathway in the lowest excited state of a cyclophane molecule can be rationalized by quantitatively studying the CT-exciton contribution of the corresponding excited-state wavefunction in the MIM representation. Here, we adopt a simple strategy to determine the relative importance of the through-bond and through-space contributions in cyclophane by comparing the weights of CT excitons in the lowest excited state for the cyclophane under study and the hypothetical molecule with different fragmentation schemes as discussed in previous section. The results of calculations using the PPP and INDO Hamiltonians for excited states and the AM1 and DFT methods for the ground-state geometry are shown in Table 1. For the molecule 1 (entry 1 in Table 1), we can see that the contribution of the CT exciton to the lowest excited state is close to that of the same molecule with the double-bond tethers removed. This can easily be understood because p-orbitals on tethers are almost perpendicular to those on benzene moieties. More careful examination shows that the weight of the CT exciton diminishes slightly with the introduction of the tethers due to the increasing of conjugation length at the chain end for the two chromophores in the cyclophane, and therefore the
TABLE 1: Relative Contributions of CT Exciton to the Lowest Excited State of Cyclophandiene 1-4

\begin{tabular}{ccccc}
\hline entry & MIM & $\begin{array}{c}\text { no tether } \\
(\mathrm{CT} \%)\end{array}$ & $\begin{array}{c}\text { tether } \\
(\mathrm{CT} \%)\end{array}$ & $\begin{array}{c}\text { T-space:T-bond } \\
(\%)\end{array}$ \\
\hline 1 & $\mathbf{1}$ (AM1) $^{a}$ & 27.79 & 27.04 & $100: 0$ \\
2 & $\mathbf{2}$ (AM1) $^{a}$ & 6.32 & 5.22 & $100: 0$ \\
3 & $\mathbf{3}$ (AM1) $^{a}$ & 0.14 & 19.65 & $1: 99$ \\
4 & $\mathbf{4}(\mathrm{AM} 1)^{a}$ & 0.99 & 4.41 & $22: 78$ \\
5 & $\mathbf{4}(\mathrm{DFT})^{a}$ & 0.60 & 6.85 & $9: 91$ \\
6 & $\mathbf{4}(\mathrm{AM} 1)^{b}$ & 0.74 & 2.51 & $29: 71$ \\
7 & $\mathbf{4}(\mathrm{DFT})^{b}$ & 0.46 & 4.87 & $9: 91$ \\
8 & $\mathbf{4}(\mathrm{AM} 1)^{c}$ & 0.65 & 3.12 & $21: 79$ \\
9 & $\mathbf{4}(\mathrm{DFT})^{c}$ & 0.36 & 4.52 & $8: 92$
\end{tabular}

${ }^{a}$ MIM/PPP (Full SCI) model. ${ }^{b}$ MIM/PPP (four state) model. ${ }^{c}$ MIM/ INDO(S) (four state) model.

excitonic interaction is reduced. We could reasonably suggest that molecule 1 belongs to the through-space case according to relative CT-exciton contributions. This is in agreement with the conclusions deduced from the NLO studies in which cyclophanes derived from the [2.2]paracyclophane are shown to be through-space delocalization. ${ }^{8}$

For cyclophane 2 consisting of two para-connected teraryls with or without tethers removed, as shown in the entry 2 of Table 1, the CT-exciton contributions to the lowest excited state decreased significantly due to the increasing chromophore length. Similar to that for molecule $\mathbf{1}$, the through-space pathway is still the dominant channel for delocalization because this molecule also has the near cofacial arrangement. However, molecule $\mathbf{3}$ with meta-connected moieties exhibits a significant CT-exciton contribution only when the tether was attached and, thus, this molecule can be regarded as belonging to the throughbond delocalization. For molecule 4 , the contribution of CT exciton for situation without tethers is also very small just like that of the molecule $\mathbf{3}$, whereas the weight of CT exciton can be enhanced almost 5-fold by introducing the double-bond tethers. This means that the presence of tethers could significantly increase the electronic communication between two chromophores in the cyclophane. The calculated CT exciton contribution based on the DFT-optimized geometry of molecule 4 shows a qualitatively similar result, except that the relative through-bond contribution is about $10 \%$ larger (entry 5) with respect to AM1 results (entry 4). This feature is owing to the smaller dihedral angle of the DFT geometry of $\mathbf{4}$. Although these two structures are slightly different, the data indicate that molecule $\mathbf{4}$ is a case of through-bond delocalization.

We also examined the effect of the number of orbitals $(n)$ included in the MIM CI matrix on the nature of delocalization pathway for molecule $\mathbf{4}$ at two different optimized geometries using AM1 and DFT methods. The percentage of the throughbond delocalization for molecule $\mathbf{4}$ at AM1-optimized geometry is quite sensitive to the number of orbitals included as shown in Figure 1. The through-bond contribution changes from 35\% to $77 \%$ as the number of orbitals included is changed from 4 to 36. It is worthy to note that, for the AM1 geometry, the truncated model with only four orbitals, the through-bond contribution is smaller than that of through-space, whereas in the situation with more than twelve orbitals included, the through-bond channel becomes the dominant delocalization pathway. Alternatively, the through-bond contribution changes slightly from $82 \%$ to $91 \%$ with the increasing number of orbitals for the optimized geometry by the DFT method, which might be due to the fact that the DFT-optimized geometry shows a slightly planar structure $\left(40^{\circ}\right)$ compared to that by the AM1 calculation $\left(45^{\circ}\right)$.

Scope of Truncated Four-State MIM Model. Previously, we proposed a four-state MIM model parametrized by supermo- 


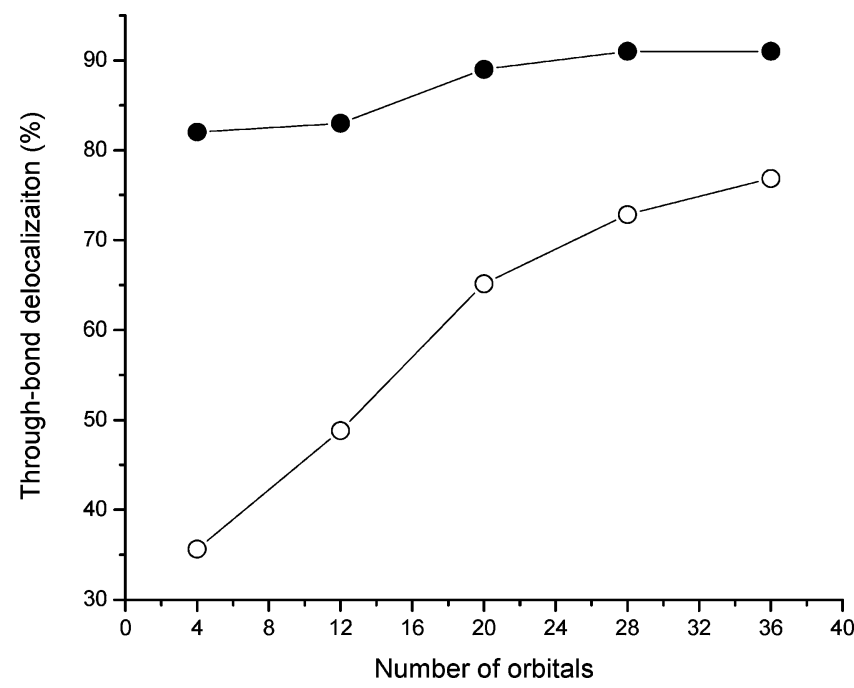

Figure 1. Various truncated MIM model for molecule $\mathbf{4}$ with different optimized geometries open circles for AM1 geometry and closed circles for DFT geometry.

(a)

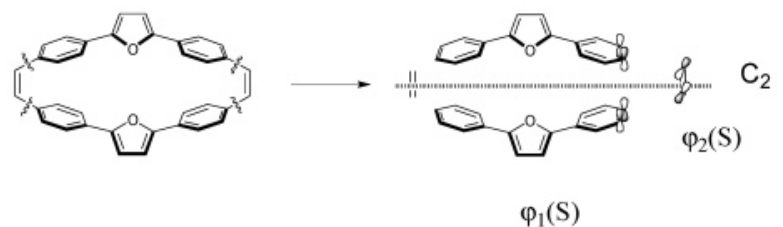

(b)
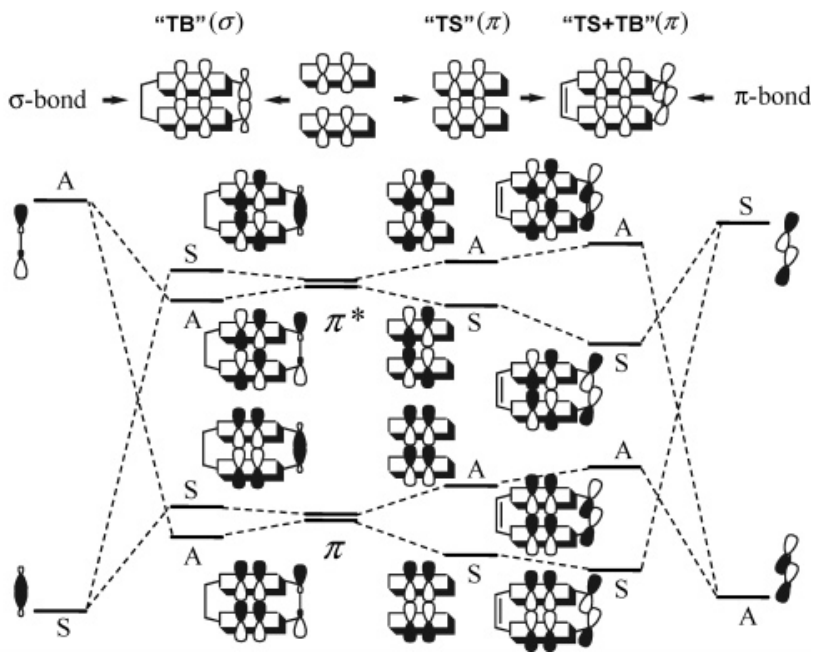

Figure 2. Orbital diagram for "through-space" and "through-bond" interaction in the class I/II cyclophanedienes with $C_{2}$ symmetry element.

lecular calculations such as PPP or INDO/S with SCI scheme. The critical information of the parametrized treatment other than SCI results is to obtain electron- and hole-transfer integrals by half of the splitting of the HOMO and LUMO levels of two monomers. Here, we will discuss the orbital interaction by disconnection of the molecule $\mathbf{4}$ to two terayl chromophores and two tethers as given in Figure 2a. First, we consider two noninteracting identical teraryl monomers without tethering double bonds. In the absence of any through-space $\pi-\pi$ interactions, the two HOMOs (LUMOs) of the two monomers are degenerate in energy. When the two monomers were allowed to interact, the HOMOs (LUMOs) would be split into two levels as shown in Figure 2b. In general, the splitting energy for
HOMOs is generally larger than that of LUMOs, which can be rationalized by the number nodal planes in these MOs. Therefore, through-space $\pi-\pi$ interactions would lead to a holetransfer integral larger than the electron-transfer integral. In the following, the origin of the orbital interaction and molecular symmetry will be discussed. Because molecule $\mathbf{4}$ belongs to the $C_{2 h}$ point group, the rotation about the 2 -fold axis, which interchanges two fragment molecular orbitals, can be used to classify the delocalized molecular orbitals. ${ }^{32}$ Now, we consider $\pi$ orbitals on two teraryl chromophores. After the $C_{2}$ rotation is operated on the delocalized molecular orbital, the symmetry symbol of this orbital is $\mathbf{A}$ when the phase of the orbital changed and the other situation is $\mathbf{S}$. Accordingly, the four frontier energy levels with through-space $\pi-\pi$ interactions, are classified as ASAS symmetry in the order of descending energy. Second, we include the tether portions, and it is reasonable to single out the essential $\sigma$ and $\pi$ orbitals without considering the $\sigma$ bonds between tethers and chromophores. ${ }^{32}$ The symmetries of the HOMO and LUMO of the $\pi$ bond in the tether are $\mathbf{A}$ and $\mathbf{S}$, respectively. According to the symmetry constraint, the HOMO (A) of the $\pi$ bond in the tether would interact with the $\mathrm{HOMO}(\mathbf{A})$ and LUMO+1 (A) of the chormophore portion and also the LUMO (S) of the $\pi$ bond in the tether can interact with the LUMO (S) and HOMO-1 (S) of the chromophroes. It is noteworthy that the $\mathbf{S S} \pi-\pi$ interaction revealed bonding/ constructive as shown in Figure 2. According to perturbation theory, the SS interaction between LUMO of the $\pi$-bond tethers and the LUMO of the chrompores would be larger than that of HOMO -1 because of energy difference of the energy levels. As a result, the splitting of the LUMO and LUMO+1 would be enhanced by through-bond $(\pi)$ interaction. Note that after allowing the through-bond $\pi-\pi$ interaction, the energy levels would remain the same symmetry order as those of throughspace. Therefore, both through-space $(\pi-\pi)$ and though-bond $(\pi-\pi)$ interaction will increase the splitting energy and this character might be used for the estimation of the electron- and hole-transfer integrals. Otherwise, if any, the through-bond ( $\sigma-$ $\pi$ ) interaction is considered, the symmetry symbols of these orbitals are SASA in the order of descending energy. This feature may reduce the estimated transfer integrals, and the MIM four-state model would not work well when the $\sigma-\pi$ interaction is significant because of underestimation of the transfer integrals. The truncated four-state model provides a reasonably connection compared with MIM/PPP (Full SCI) method as shown in Table 1. The parameters for the four-state model is based on PPP and INDO/S Hamiltonian with a SCI scheme, and both the AM1 and DFT geometries of $\mathbf{4}$ show reasonable relative CT excitons (entry 6-9 in Table 1). These results indicate the nature of the delocalization in the lowest excited state of molecule $\mathbf{4}$ is mainly due to through-bond pathway. This truncated model gives a good agreement with MIM/PPP (Full SCI) method, which is according to correctly estimate electron- and hole-transfer integrals. The MIM/INDO(S) four-state model gives consistent results compared with MIM/PPP (Full SCI) method which indicates that the through-bond $(\sigma-\pi)$ contribution to the excited state is negligible in molecule 4. It is noteworthy that the comparable results of MIM/INDO(S) and MIM/PPP (Full SCI) calculations are cross reference. In other words, $\pi$-based MIM/PPP (Full SCI) model as well as the truncated four-state model might be good enough for determining the delocalization pathway in the lowest excited state.

The further details of the electronic structures of compounds 1-4 can be analyzed by viewing the MIM/CI matrices using contour plots as shown in Figure 3. For clarity, we have taken 

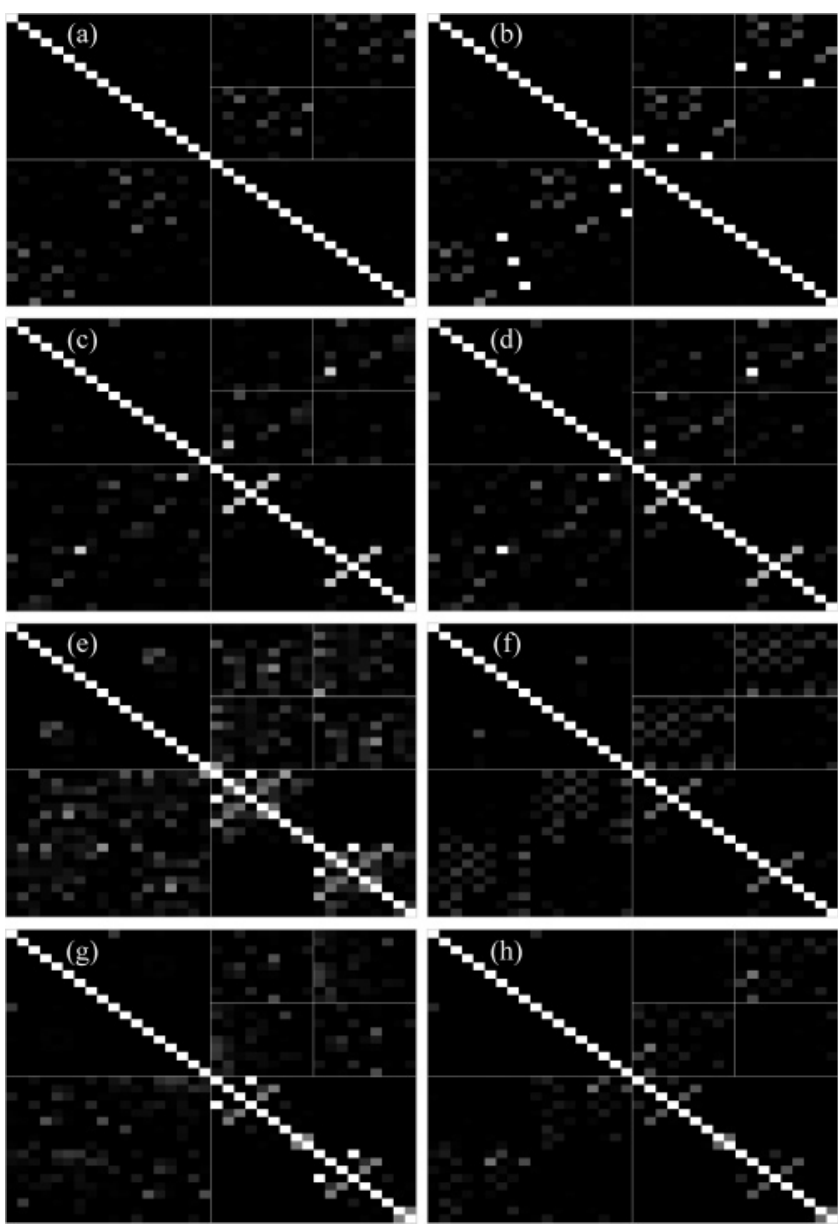

Figure 3. Contour plots of MIM matrices for compounds 1-4 (left), and same compounds with tethers removed (right).

six localized molecular orbitals from each moiety to build up the contour plot for each compound. The contour plots (Figure $3 a, b)$ of molecule $\mathbf{1}$ with or without tethers reveal similar patterns - the hole-transfer integrals are much larger than electron-transfer integrals, which can be explained by the fact that the LUMO occupied by an electron has more nodal planes and thus has stronger cancellation. A similar pattern in contour plots (Figure 3c,d) was also found for compound 2, where the hole-transfer integrals are also larger than the electron-transfer integrals, indicating that the through-space interaction is the major cause for the excited-state delocalization. Unlike molecules $\mathbf{1}$ and $\mathbf{2}$, the molecules $\mathbf{3}$ and $\mathbf{4}$ show quite different patterns in their contour plots with and without tethers. For molecule 3, meta-cyclophanediene, without the tethers, the through-space pattern in the contour plot (Figure 3f) is reproduced as indicated by the dominant hole-transfer coupling; however, there is significant enhancement in the electron-transfer interaction when the tethers are added (Figure 3e). Similarly, the electron-transfer integrals also become important in molecule 4 with tethers added as shown in Figure $3 \mathrm{~g}$,h, implying that the through-bond interaction plays the major role for cyclophanedienes with meta-linkages, which is consistent with the result based on the CT weight as shown in the Figure 1. Therefore, in additional to the calculation based on the truncated orbital MIM model, the pattern change in detailed contour plots may provide extra information for determining the through-bond or through-space delocalization pathway in the excited states.

In summary, we have applied the "molecule-in-molecule" technique under either the full SCI or four-state approximation to resolve the through-space and through-bond problems in the cyclophanediene systems. We found that the excited-state delocalization of the model cyclophanediene $\mathbf{1}$ and $\mathbf{2}$ is originated from the through-space interaction, and delocalization of molecules $\mathbf{3}$ and $\mathbf{4}$ is via the through-bond interaction. We believe that the approach proposed in this article can be applied not only to cyclophanediene systems but also to any bichromophoric systems.

Acknowledgment. This work is supported by the National Science Council and the National Taiwan University of the Republic of China.

Supporting Information Available: Derivation details of the molecule wave functions and a table of four-state matrix elements. This material is available free of charge via the Internet at http://pubs.acs.org.

\section{References and Notes}

(1) Jenekhe, S. A.; Osaheni, J. A. Science 1994, 265, 765.

(2) Gleiter, R.; Hopf, H. Modern Cyclophane Chemistry (WileyVCH: Weinheim 2004).

(3) (a) Oldham, W. J., Jr.; Miao, Y.-J.; Lachicotte, R. J.; Bazan, G. C J. Am. Chem. Soc. 1998, 120, 419. (b) Bazan, G. C.; Oldham, W. J., Jr. Lachicotte, R. J.; Tretiak, S.; Chernyak, V.; Mukamel, S. J. Am. Chem. Soc. 1998, 120, 9188. (c) Wang, S.; Bazan, G. C.; Tretiak, S.; Mukamel, S. J. Am. Chem. Soc. 2000, 122, 1289. (d) Sakai, T.; Satou, T.; Kaikawa T.; Takimiya, K.; Otsubo, T.; Aso, Y. J. Am. Chem. Soc. 2005, 127, 8082.

(4) (a) Hong, J. W.; Benmansour, H.; Bazan, G. C. Chem. Eur. J. 2003 , 9, 3192. (b) Hong, J. W.; Gaylord, B. S.; Bazan, G. C. J. Am. Chem. Soc. 2002, 124, 11868 .

(5) (a) Williams, R. V.; Edwards, W. D.; Mitchell, R. H.; Robinson, S. G. J. Am. Chem. Soc. 2005, 127, 16207. (b) Mitchell, R. H.; Brkic, Z.; Sauro, V. A.; Berg, D. J. J. Am. Chem. Soc. 2003, 125, 7581. (c) Mitchell, R. H.; Ward, T. R.; Chen, Y.; Wang, Y.; Weerawarna, S. A.; Dibble, P. W.; Marsella, M. J.; Almutairi, A.; Wang, Z.-Q. J. Am. Chem. Soc. 2003, 125, 2974. (d) Williams, R. V.; Armantrout, J. R.; Twamley, B.; Mitchell, R. H.; Ward, T. R.; Bandyopadhyay, S. J. Am. Chem. Soc. 2002, 124, 13495.

(6) (a) Woo, H. Y.; Hong, J. W.; Liu, B.; Mikhailovsky, A.; Korystov, D.; Bazan, G. C. J. Am. Chem. Soc. 2005, 127, 820. (b) Woo, H. Y.; Korystov, D.; Mikhailovsky, A.; Nguyen, T.-Q.; Bazan, G. C. J. Am. Chem. Soc. 2005, 127, 13794. (c) Bartholomew, G. P.; Rumi, M.; Pond, S. J. K.; Perry, J. W.; Tretiak, S.; Bazan, G. C. J. Am. Chem. Soc. 2004, 126, 11529.

(7) (a) Nelsen, S. F.; Konradsson, A. E.; Telo, J. P. J. Am. Chem. Soc. 2005, 127, 920. (b) Yan, X. Z.; Pawlas, J.; Goodson, T., III; Hartwig, J. F. J. Am. Chem. Soc. 2005, 127, 9105.

(8) (a) Bartholomew, G. P.; Ledoux, I.; Mukamel, S.; Bazan, G. C.; Zyss, J. J. Am. Chem. Soc. 2002, 124, 13480. (b) Zyss, J.; Ledoux, I.; Volkov, S.; Chernyak, V.; Mukamel, S.; Bartholomew, G. P.; Bazan, G. C. J. Am. Chem. Soc. 2000, 122, 11956.

(9) Lin, H.-C.; Lin, W.-Y.; Bai, H.-T.; Chen, J.-H.; Jin, B.-Y.; Luh, T.-Y. Angew. Chem., Int. Ed. 2007, 46, 897.

(10) Lin, H.-C.; Hsu, J.-H.; Lee, C.-K.; Tai, O. Y.-H.; Wang, C.-H.; Chou, C.-M.; Wu, Y.-L.; Luh, T.-Y. submitted.

(11) Beljonne, D.; Cornil, J.; Silbey, R.; Millié, P.; Brédas, J. L. J. Chem. Phys. 2000, 112, 4749 .

(12) Longuet-Higgins, H. C.; Murrell, J. N. Proc. Phys. Soc. A 1955, 68,601

(13) Warshel, A.; Parson, W. W. J. Am. Chem. Soc. 1987, 109, 6143.

(14) Parson, W. W.; Creighton, S.; Warshel, A. J. Am. Chem. Soc. 1989, 111,4277

(15) Fleischhauer, J; Ho1weler, U.; Spanget-Larsen, J.; Raabe, G; Michl, J. J. Phys. Chem. A 2004, 108, 3225.

(16) (a) Harcourt, R. D.; Scholes, G. D.; Ghiggino, K. P. J. Chem. Phys 1994, 101, 10521. (b) Hennebicq, E.; Pourtois, G.; Scholes, G. D.; Herz, L. M.; Russell, D. M.; Silva, C.; Setayesh, S.; Grimsdale, A. C.; Mullen, K.; Bredas, J.-L.; Beljonne, D. J. Am. Chem. Soc. 2005, 127, 4744.

(17) (a) Electron Transfer in Chemistry; Balzani, V., Ed.; Wiley-VCH: Weinheim, 2001. (b) Electron Transfer: From Isolated Molecules to Biomolecules, Adv. Chem. Phys.; Bixon, M., Jortner, J., Eds.; Wiley: New York, 1999; Vols. 106-107. (c) Newton, M. D. Chem. Rev. 1991, 91, 767. (d) Brédas, J. L.; Beljonne, D.; Coropceanu, V.; Cornil, J. Chem. Rev. 2004, 104, 4971. (e) Li, X. Y.; Tang, X. S.; He, F. C. Chem. Phys. 1999, 248, 137. (f) Li, X. Y.; He, F. C. J. Comput. Chem. 1999, 20, 597.

(18) Koopmans, T. Physica 1934, 1, 104.

(19) Coropceanu, V.; Cornil, J.; Da Silva Filho, D. A.; Olivier, Y.; Silbey, R.; Brédas, J. L. Chem. Rev. 2007, 107, 926. 
(20) (a) Blancafort, L.; Voityuk, A. A. J. Phys. Chem. A 2006, 110, 6426. (b) Pati, R.; Karna, S. P. J. Chem. Phys. 2001, 115, 1703. (c) RodriguezMonge, L.; Larsson, S. J. Phys. Chem. 1996, 100, 6298.

(21) Dewar, M. J. S.; Zoebisch, E. G.; Healy, E. F.; Stewart, J. J. P. J. Am. Chem. Soc. 1985, 107, 3902.

(22) (a) Lofthagen, M.; Chadha, R.; Siegel, J. S. J. Am. Chem. Soc. 1991, 113, 8785-8790. (b) Tobe, Y.; Kawaguchi, M.; Kakiuchi, K.; Naemura, K. J. Am. Chem. Soc. 1993, 115, 1173-1174. (c) Srinivasan, M.; Sankararaman, S.; Dix, I.; Jones, P. G. Org. Lett. 2000, 2, 3849-3851.

(23) Lee, C.; Yang, W.; Parr, R. G. Phys, Rev. B 1988, 37, 785. (b) Becke, A. D. Phys. Rev. A 1988, 38, 3098. (c) Becke, A. D. J. Chem. Phys. 1993, 98, 5648 .

(24) (a) Ditchfield, R.; Hehre, W. J.; Pople, J. A. J. Chem. Phys. 1971 54, 724. (b) Hehre, W. J.; Ditchfield, R.; Pople, J. A. J. Chem. Phys. 1972, 56, 2257. (c) Hariharan, P. C.; Pople, J. A. Mol. Phys. 1974, 27, 209. (d) Gordon, M. S. Chem. Phys. Lett. 1980, 76, 163. (e) Hariharan, P. C.; Pople J. A. Theor. Chim. Acta 1973, 28, 213.
(25) Walden, S.; Glatzhofer, D. T. J. Phys. Chem. A 1997, 101, 8233 (26) Hensler, D.; Hohlneicher, G. J. Phys. Chem. A 1998, 102, 10828.

(27) Szabo, A.; Ostlund, W. S. Modern Quantum Chemistry: Introduction to Advanced Electronic Structure Theory (Macmillan, New York 1982).

(28) Pople, J. A.; Beveridge, D. L.; Dobosh, P. A. J. Chem. Phys. 1967, 47, 2026.

(29) Zerner, M. C.; Loew, G. H.; Kichner, R. F.; Mueller-Westerhoff, U. T. J. Am. Chem. Soc. 1980, 102, 589.

(30) Zojer, E.; Cornil, J.; Leising, G.; Brédas, J. L. Phy. Rev. B 1999, $59,7957$.

(31) Cornil, J.; Vanderdonckt, S.; Lazzaroni, R.; dos Santos, D. A.; Thys, G.; Geise, H. J.; Yu, L.-M.; Szablewski, M.; Bloor, D.; Lögdlund, M. Salaneck, W. R.; Gruhn, N. E.; Lichtenberger, D. L.; Lee, P. A.; Armstrong, N. R.; Brédas, J. L. Chem. Mater. 1999, 11, 2436-2443.

(32) (a) Hoffmann, R. Acc. Chem. Res. 1971, 4, 204. (b) Hoffmann, R.; Imamura, A.; Hehre, W. J. J. Am. Chem. Soc. 1968, 90, 1499. 